\title{
DEDEKIND COMPLETENESS AND A FIXED-POINT THEOREM
}

\author{
E. S. WOLK
}

1. Introduction. McShane $(\mathbf{5 , 6})$ has introduced the concept of "Dedekind completeness" for partially ordered sets, which seems to be a natural generalization of the usual concept of completeness for lattices. It is the purpose of this paper to discuss some of the properties of Dedekind completeness, particularly with respect to a rather natural class of partially ordered sets which we call "uniform." Among our results we obtain an analogue of MacNeille's "completion by cuts." We also extend the well-known fixed-point theorem, due to Tarski (7), and then generalize the characterization of a complete lattice due to Davis (3).

2. Dedekind completeness. Let $P$ be a partially ordered set (poset) with respect to a relation $\leqslant$. We assume that $P$ has a greatest element $I$ and a least element $O$.

Definition 1 . We say that a set $S \subset P$ is up-directed if and only if for each $a \in S, b \in S$, there exists $c \in S$ with $a \leqslant c, b \leqslant c$. Dually, $S$ is downdirected if and only if for each $a \in S, b \in S$, there exists $c \in S$ with $c \leqslant a$, $c \leqslant b$.

Thus, any subset of $P$ which has a greatest element is up-directed, and dually. The following definition is essentially that of McShane.

Definition 2. A poset $P$ is Dedekind complete if and only if every updirected subset of $P$ has a least upper bound in $P$ and every down-directed subset has a greatest lower bound in $P$.

Example 1. It is clear that the concepts of Dedekind completeness and ordinary completeness coincide if $P$ is a lattice. A simple example of a Dedekind complete poset, which is not a lattice, is provided by the set $C$ of all closed disks in the Euclidean plane $E_{2}$, partially ordered by set inclusion, and with $O$ and $I$ elements adjoined. To show that $C$ is Dedekind complete, let $A$ be an up-directed subset of $C$, and let

$$
X=\left\{x \mid x \in E_{2} \text { and } x \in a \text { for some } a \in A\right\} .
$$

If $X$ is an unbounded subset of $E_{2}$, then clearly l. u. b. $(A)=I$. If $X$ is bounded choose two points $x$ and $y$ in the closure of $X$ such that the distance from $x$ to $y$ is equal to the diameter of $X$. Let $m$ be a closed disk with the line seg-

Received January 12, 1956. 
ment connecting $x$ and $y$ as its diameter. Straightforward arguments then show that

(i) no point of $X$ is exterior to $m$, and

(ii) every interior point of $m$ is a point of $X$. Thus $m=1$. u. b. $(A)$. The obvious dual argument will then show that any down-directed subset of $C$ has a g. 1. b.

If $A \subseteq P$, let

$$
\begin{aligned}
& A^{*}=\{x \mid x \in P \text { and } x \geqslant a \text { for all } a \in A\}, \\
& A^{+}=\{x \mid x \in P \text { and } x \leqslant a \text { for all } a \in A\} .
\end{aligned}
$$

We shall write $A^{*+}$ for the set $\left(A^{*}\right)^{+}$. We shall make important use of the following concept:

Definition 3. A poset $P$ is uniform if and only if $A^{*}$ is a down-directed set for every up-directed subset $A$, and dually, $B^{+}$is up-directed for every down-directed subset $B$.

Any lattice is obviously a uniform poset. As an example of a uniform poset, which is not a lattice and not Dedekind complete, we may take the set of all closed disks in the plane with rational radii, partially ordered by set inclusion, and with $O$ and $I$ elements adjoined.

We have the following trivial lemma:

Lemma 1. A uniform poset $P$ is Dedekind complete if and only if every updirected subset of $P$ has a l.u.b. in $P$ (or every down-directed subset of $P$ has a g. 1. b. in $P$ ).

We shall also use a strong form of Zorn's lemma due to Bourbaki (2):

Lemma (Bourbaki). If every well-ordered chain in a poset $S$ has an upper bound in $S$, then $S$ has a maximal element.

As a consequence of the above lemma the reader may easily deduce

Lemma 2. If $Z$ is any chain in a poset $P$, then there exists a well-ordered chain $C \subset Z$ with $C^{*}=Z^{*}$.

We now have the following theorem:

Theorem 1. A poset $P$ is Dedekind complete if and only if $P$ is uniform and every well-ordered chain in $P$ has a 1 . u. b.

Proof. If $P$ is Dedekind complete, and $S$ is up-directed in $P$, then $S^{*}$ has a least element and hence is down-directed. The obvious dual statement also holds: thus $P$ is uniform and the conclusion follows. Conversely, let $P$ be uniform and suppose that every well-ordered chain in $P$ has a 1. u. b. Let $A$ be any down-directed subset of $P$, and let $Z$ be a maximal chain in $A^{+}$. We assert that $Z$. has a 1 . u. b., $m$, for otherwise Lemma 2 would provide us with a contradicticn of our hypothesis. If $a \in A$, we have $a \geqslant z$ for all $z \in Z$; 
hence $a \geqslant m$ and $m \in A^{+}$. By maximality of $Z, m$ is a maximal element of $A^{+}$. We assert that $m$ is the greatest element of $A^{+}$. For suppose that there exists $c \in A^{+}$with $c>m$. Since $A^{+}$is up-directed, there exists $x \in A^{+}$with $x \geqslant m, x \geqslant c$, contradicting the maximality of $m$. Thus $m=$ g. 1 . b. $(A)$, and $P$ is Dedekind complete by Lemma 1.

As a corollary we have the following known result, for which a proof seems to have thus far been lacking in the literature:

Corollary. A lattice $L$ is complete if and only if every well-ordered chain in L has a 1. u. b.

Let us call a chain $Z$ in $P$ inversely well-ordered if and only if every subset of $Z$ has a greatest element. We then have obvious dual formulations of Lemma 2 and Theorem 1. We shall also need the following lemma, which extends a result of Davis (3, Lemma 1, p. 311); our proof of it becomes trivial by employing Zorn's lemma (rather than transfinite induction as in (3)) :

Lemma 3. Let $P$ be a uniform poset, and let $Z$ be an inversely well-ordered chain in $P$ with no g. l. b. in $P$. Then there exists a well-ordered chain $Y$ in $P$ such that

(i) $y \in Y$ implies $y<z$ for all $z \in Z$, and

(ii) $Y^{*} \cap Z^{+}$is empty.

Proof. $Z^{+}$is up-directed, by our hypothesis of uniformity; hence $Z^{+}$has no maximal elements. Then by the lemma of Bourbaki there exists a well-ordered chain $Y$ in $Z^{+}$such that $Y^{*} \cap Z^{+}$is empty.

3. Imbedding of a uniform poset in a Dedekind complete poset. We shall now obtain an analogue of MacNeille's well-known imbedding of a poset in a complete lattice (4; also see 1, p. 58).

Definition 4. A subset $J$ of a poset $P$ is a normal ideal in $P$ ("closed ideal" in the terminology of Birkhoff) if and only if $J^{*+}=J$. A subset of $P$ of the form

$$
J_{a}=\{x \mid x \in P \text { and } x \leqslant a\}
$$

is called a principal ideal.

Lemma 4. A subset of $P$ is a normal ideal if and only if it is the intersection of a set of principal ideals (cf. 1; p. 62, problem 4).

Proof. Let $S \subset P$ and let

$$
1=\bigcap_{x \in S} J_{x}
$$

Then $A=S^{+}$. In general we have $S \subset S^{+*}$; hence $S^{+} \supset\left(S^{+*}\right)^{+}$, or $A \supset A^{*+}$. Since in general $A \subset A^{*+}$, it follows that $A$ is a normal ideal. Conversely, if $A$ is a normal ideal in $P$, then

$$
A=\left(A^{*}\right)^{+}=\bigcap_{x \in A^{*}} J_{x}
$$


For uniform posets we now have another characterization of Dedekind completeness, which generalizes a known result for complete lattices (1; p. 59, exercise 2):

Theorem 2. A uniform poset $P$ is Dedekind complete if and only if every updirected normal ideal in $P$ is principal.

Proof. Let $P$ be Dedekind complete, and let $J$ be an up-directed normal ideal in $P$. Then $J$ has a 1. u. b. $m$, and $m \in J^{*+}=J$. It follows that $J$ is principal. To prove the converse, let $A$ be a down-directed subset of $P$. By Lemma 4,

$$
A^{+}=\bigcap_{a \in A} J_{a}
$$

is a normal ideal, which by hypothesis is up-directed. Hence $A^{+}$has a 1. u. b., which is the g. 1 . b. of $A$. Thus $P$ is Dedekind complete by Lemma 1 .

Now let $N(P)$ be the set of all up-directed normal ideals of $P$, partially ordered by inclusion. The correspondence $x \leftrightarrow J_{x}$ is a one-to-one orderpreserving mapping of $P$ into a subset of $N(P)$. Furthermore, we have

Theorem 3. If $P$ is a uniform poset, then $N(P)$ is Dedekind complete.

Proof. Let $\Sigma$ be an up-directed subset of $N(P)$, and let

$$
A=\bigcup_{J \in \Sigma} J
$$

(where $\mathrm{U}$ denotes set union). It is easily seen that $A$ is an up-directed subset of $P$. Hence $A^{*}$ is down-directed, and $A^{*+}$ is up-directed. Since $A^{*+}$ is the smallest normal ideal containing $A$, we have $A^{*+}=1$ u. b. $(\Sigma)$. Now let $\Omega$ be a down-directed subset of $N(P)$. We first show that

$$
B=\bigcup_{\in \in \Omega} J^{*}
$$

is a down-directed subset of $P$. Let $a$ and $b$ be arbitrary elements of $B$; then there exist $J_{1}, J_{2} \in \Omega$ with $a \in J_{1}{ }^{*}, b \in J_{2}{ }^{*}$. By our hypothesis on $\Omega$, there exists $J_{3} \in \Omega$ with $J_{3} \subset J_{1} \cap J_{2}$. Then $J_{3}{ }^{*} \supset\left(J_{1} \cap J_{2}\right)^{*} \supset J_{1}{ }^{*} \cup J_{2}{ }^{*}$. But $J_{3}{ }^{*}$ is down-directed, by uniformity of $P$ : hence there exists $c \in J_{3}{ }^{*}$ with $c \leqslant a, c \leqslant b$, and thus $B$ is down-directed. Now let

$$
K=\bigcap_{J \in \Omega} J
$$

But

$$
\bigcap_{J \in \Omega} J=\bigcap_{J \in \Omega} J^{*+}=\left(\bigcup_{J \in \Omega} J^{*}\right)^{+}=B^{+} .
$$

Hence $K$ is an up-directed normal ideal, and $K=$ g. 1. b. $(\Omega)$.

Example 2. Let $P$ be the set of all closed disks in the plane with rational radii, ordmed by inclusion. If $z$ is an arbitrary closed disk in the plane, then the set

$$
S(z)=\{a \mid a \in P \text { and } a \subset z\}
$$


is an up-directed normal ideal in $P$; and conversely, the reader may verify that every such ideal is of the form $S(z)$ for some disk $z$. Hence the "Dedekind completion" $N(P)$ is isomorphic to the set of all closed disks in the plane.

Example 3. If $P$ is not uniform, then $N(P)$ may fail to be Dedekind complete. We construct an example of such a poset $P$ as follows. Let $A=\left\{a_{i j}\right\}$ $(i=1,2, \ldots ; j=1,2, \ldots)$ be an infinite rectangular array, in which $i$ denotes the column index, $j$ the row index. We partially order $A$ by defining $a_{i j}<a_{m n}$ if and only if $i<m$ or $j<n$. We surmount this array with a sequence $\left\{z_{i}\right\}$ of mutually incomparable elements (with respect to our ordering) such that $a_{i j}<z_{i}$ for each $i$ and each $j$. We adjoin two more incomparable elements $x$ and $y$ which are upper bounds for the set $\left\{z_{i}\right\}$. We then let $P$ be the set consisting of the array $A=\left\{a_{i j}\right\}$, the set $\left\{z_{i}\right\}$, the elements $x$ and $y$, and $O$ and $I$ elements; and let $P$ be partially ordered as described above. Thus we have $a_{i j}<z_{k}$ if and only if $i \leqslant k$. We see that $A$ is an up-directed subset of $P$. (Note, however, that $A^{*+}$ is the union of $A$ and the set $\left\{z_{i}\right\}$, and hence is not up-directed. Thus $A^{*+}$ is not an element of $\left.N(P)\right)$. Hence

$$
\Sigma=\left\{J_{a} \mid a \in A\right\}
$$

is an up-directed subset of $N(P)$. But the set $\Sigma^{*}$ contains $J_{x}$ and $J_{y}$ as minimal elements; hence $\Sigma$ has no l. u. b. in $N(P)$.

4. The fixed-point theorem. If $f$ is a function mapping a poset $P$ into itself, we say that $f$ is isotone if and only if $x \leqslant y$ implies $f(x) \leqslant f(y) . x$ is a fixed-point of $f$ if and only if $x=f(x)$. For any isotone function $f$ on $P$ let us write $H(f)=\{x \mid x \in P$ and $x \leqslant f(x)\}$.

Definition 5. An isotone function $f$ on a poset $P$ is directable if and only if $H(f)$ is an up-directed subset of $P$.

The reader may verify that any isotone function on a lattice is directable. Thus the following theorem generalizes the fixed-point theorem of Tarski (7, Theorem 1):

THEOREM 4. If every up-directed subset of a poset $P$ has a 1. u. b. in $P$, then every directable function on $P$ has a fixed-point.

Proof. Let $f$ be a directable function on $P$ and let $u=1$. u. b. $[H(f)]$. We easily prove, precisely as in the proof of Theorem 1 of (7), that $u$ is a fixedpoint of $f$. We omit the details.

We now obtain a generalization of the result of Davis (3, Theorem 2) :

THEOREM 5. If every directable function on a uniform poset $P$ has a fixedpoint, then $P$ is Dedekind complete.

Proof. Assume $P$ is not Dedekind complete. Applying the dual formulation of Theorem 1 and then Lemma 3, we infer that there exist two chains $Y$ and $Z$ in $P$ such that 
(i) $Y$ is well-ordered and $Z$ is inversely well-ordered,

(ii) $y \in Y$ implies $y<z$ for all $z \in Z$,

(iii) $Y^{*} \cap Z^{+}$is empty.

We shall proceed to obtain a contradiction by defining a directable function $f$ on $P$ which has no fixed-points. We do this exactly as in (3, pp. 313-314). To define $f\left(x_{0}\right)$ for an arbitrary $x_{0} \in P$ we distinguish two cases:

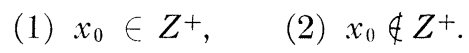

In case (1) we have $x_{0} \notin Y^{*}$. Let $Y\left(x_{0}\right)=\left\{y \mid y \in Y\right.$ and $\left.y>x_{0}\right\} . \quad Y\left(x_{0}\right)$ has a least element $y_{0}$, which we define as $f\left(x_{0}\right)$. In case $(2)$, let $Z\left(x_{0}\right)=$ $\left\{z \mid z \in Z\right.$ and $\left.z<x_{0}\right\} . Z\left(x_{0}\right)$ has a greatest element $z_{0}$, which we define as $f\left(x_{0}\right)$. It is clear that $f$ can have no fixed-points. The proof that $f$ is isotone is identical with that in (3, p. 314): we therefore omit the details. It remains to show that $f$ is directable. From our definition of $f$ it is clear that $x \in H(f)$ implies that $f$ falls in case (1) above; i.e., $x \in Z^{+}$. Also it is clear that $Y \subset H(f)$. Now suppose that we have $a \in H(f), b \in H(f)$. Then $a<f(a), b<f(b)$, and $f(a) \in Y, f(b) \in Y$. Let $c=\max \{f(a), f(b)\}$. We have $c>a, c>b$, and $c \in H(f)$, thus completing the proof.

Combining Theorems 4 and 5 , we obtain the following characterization of Dedekind completeness:

Corollary. A uniform poset $P$ is Dedekind complete if and only if every directable function on $P$ has a fixed-point.

\section{REFERENCES}

1. G. Birkhoff, Lattice theory, Amer. Math. Soc. Colloquium Publications, 25 (1948).

2. N. Bourbaki, Sur le théorème de Zorn, Archiv. der Math., 6 (1949-50), 434-437.

3. Anne C. Davis, A characterization of complete lattices, Pacific J. Math., 5 (1955), 311-319.

4. H. MacNeille, Partially ordered sets, Trans. Amer. Math. Soc., 42 (1937), 416-460.

5. E. J. McShane, Order-preserving maps and integration processes, Annals of Math. Studies, 31 (Princeton, 1953).

6. E. J. McShane, Partial orderings and Moore-Smith limits, Amer. Math. Monthly, 59 (1952), $1-11$.

7. A. Tarski, A lattice-theoretical fixpoint theorem and its applications, Pacific J. Math. 5 (1955), 285-309.

\section{University of Connecticut}

